\title{
Interação genótipo x ambiente e características pré-desmama em animais da raça Simental em duas estações de nascimento
}

\author{
lara Del Pilar Solar Diaz(1), Francisco Ribeiro de Araujo Neto(1), Luiz Fernando Aarão Marques(2) \\ e Henrique Nunes de Oliveira ${ }^{(1)}$
}

\begin{abstract}
(1)Universidade Estadual Paulista, Faculdade de Ciências Agrárias e Veterinárias, Departamento de Zootecnia, Via de acesso Prof. Paulo Donato Castellane, s/no, CEP 14884-900 Jaboticabal, SP. E-mail: iarasolar@hotmail.com, netozoo@hotmail.com, holiveira@fcav.unesp.br (2)Universidade Federal do Espírito Santo, Centro de Ciências Agrárias, Alto Universitário, s/nº, Guararema, CEP 29500-000 Alegre, ES. E-mail: Ifernando@cca.ufes.br
\end{abstract}

Resumo - O objetivo deste trabalho foi avaliar o efeito da interação genótipo x ambiente (GxA), nas características peso à desmama e ganho de peso do nascimento à desmama, em machos e fêmeas da raça Simental, nascidos nas estações chuvosa e seca. Foram avaliados 20 mil animais, aos 210 dias de idade. Realizou-se uma análise multicaracterística, que considerou como distinta a mesma característica nos diferentes grupos ambientais, e uma análise unicaracterística, que considerou cada característica como a mesma em todos os grupos ambientais. A interação GxA foi avaliada por meio da correlação genética $\left(\mathrm{r}_{\mathrm{g}}\right)$. As interações foram consideradas importantes quando os valores de $\mathrm{r}_{\mathrm{g}}$ ficaram abaixo de 0,80 . As distribuições posteriores das estimativas de herdabilidades mostraram ausência de heterogeneidade de variâncias entre os sexos, entretanto houve interação GxA entre os grupos ambientais. Observaram-se valores de correlação genética de 0,54 a 0,78 e 0,55 a 0,75 para peso à desmama e ganho de peso do nascimento à desmama, respectivamente. As seleções, baseadas tanto na análise unicaracterística quanto na multicaracterística, não mostraram diferenças significativas quanto ao ganho genético dos animais. Há efeito das estações de nascimento nas características avaliadas, em todos os grupos ambientais, e a interação GxA é mais evidente em fêmeas do que em machos.

Termos para indexação: correlações genéticas, fêmeas, inferência bayesiana, machos, plasticidade fenotípica.

\section{Genotype $x$ environment interaction and pre-weaning traits in Simmental animals in two birth seasons}

\begin{abstract}
The objective of this study was to evaluate the effect of the genotype $x$ environment (GxE) interaction on weaning weight and weight gain from birth to weaning, in Simmental males and females born in the rainy and dry seasons. Twenty thousand animals were evaluated at 210 days of age. A multitrait analysis was performed, in which the same feature was considered distinct for the different environmental groups; and a single trait analysis was done, in which each trait was considered as the same in all environmental groups. The GxE interaction was assessed by genetic correlation $\left(r_{g}\right)$. The interactions were considered important when $r_{g}$ values were below 0.80 . The posterior distributions of heritability estimates showed the absence of heterogeneity of the variances between sexes, although there was GxE interaction between the environmental groups. The observed values of genetic correlation were 0.54 to 0.78 and 0.55 to 0.75 for weight at weaning and weight gain from birth to weaning, respectively. The selection based both on the single trait and on the multitrait analysis showed no significant differences for genetic gain of the animals. Birth seasons have effect on the evaluated traits in all environmental groups, and the GxE interaction is more evident in females than males.
\end{abstract}

Index terms: genetic correlations, females, bayesian inference, males, phenotype plasticity.

\section{Introdução}

Para a escolha de animais geneticamente superiores na reprodução, são realizadas análises em que estimativas dos valores genéticos dos animais são obtidas por meio das informações do fenótipo. $\mathrm{O}$ fenótipo é determinado pelo genótipo, pelo ambiente e pela interação desses dois fatores. A interação genótipo $\mathrm{x}$ ambiente (GxA) é a mudança relativa no desempenho de um mesmo genótipo quando exposto a diferentes ambientes. Tanto a definição quanto o entendimento do que seria a interação GxA passaram por diversas mudanças ao longo do tempo (Jong \& Bijma, 2002; Kolmodin et al., 2002; West-Eberhard, 2003). 
Segundo Pégolo (2010), o interesse pelos efeitos da interação GxA tem aumentado, à medida que os programas de melhoramento genético tendem a aumentar sua abrangência geográfica. Assim, o efeito do sexo sobre as características do animal tem gerado discussões quanto ao melhoramento genético. Machos e fêmeas estão sujeitos a diferentes objetivos de seleção e, por essa razão são também submetidos a um tratamento diferenciado nas fazendas. A depender do ambiente, essa diferença pode aumentar, o que ressalta a importância da interação GxA, pois a avaliação genética dos animais é realizada com a consideração dos efeitos genéticos aditivos semelhantes nos dois sexos.

Não se discute a presença da interação, uma vez que existem diferenças biológicas entre os sexos dos animais, o que se procura saber é qual a extensão dessa interação nos genótipos, e quanto isso influencia o valor genético de um animal. No caso do Brasil, quando se considera a variação na qualidade das pastagens, nas distintas estações do ano, é importante avaliar de que maneira essa variação influi no desempenho de ambos os sexos.

O objetivo deste trabalho foi avaliar o efeito da interação genótipo $\mathrm{x}$ ambiente nas caracteristicas peso à desmama e ganho de peso do nascimento à desmama, em machos e fêmeas da raça Simental, nascidos nas estações chuvosa e seca.

\section{Material e Métodos}

Dados e provenientes dos arquivos da Associação Brasileira dos Criadores das raças Simental e Simbrasil (ABCRSS), referentes a 20 mil animais Simental, foram utilizados a para estimar os parâmetros genéticos necessários à determinação do efeito da interação genótipo $\mathrm{x}$ ambiente no peso à desmama (P210) e no ganho de peso do nascimento à desmama (GND), em machos (M) e fêmeas (F). Foram consideradas duas estações de nascimento: a chuvosa (C, outubro-março); e a seca (S, abril-setembro). Pela combinação das informações de sexo e estação de nascimento, foram formados quatro grupos de ambiente (GA): P210_ FC; P210_FS; P210_MC; P210_MS, quanto ao peso à desmama; e GND_FC; GND_FS; GND_MC; GND_MS, quanto ao ganho do nascimento à desmama. Para cada grupo, a característica analisada (P210 ou GND) foi considerada como uma característica diferente (Tabela 1).

O procedimento GLM ("general linear models") (SAS Institute, 2008) foi usado para auxiliar na definição dos efeitos a serem incluídos nos grupos de contemporâneos, que foram formados a partir das informações de ano, propriedade e manejo, sexo e estação de nascimento. Grupos de contemporâneos com menos de cinco observações foram retirados da análise, bem como os registros de animais com dados inconsistentes. $\mathrm{O}$ arquivo de genealogia, utilizado para montar a matriz de parentesco, continha a identificação do animal, pai e mãe, e totalizou 88.072 animais.

Tanto para P210 quanto para GND, os componentes de covariância e variância foram obtidos por meio do programa GIBBS2f90, desenvolvido por Misztal (2009), em análise de característica única, que considerou cada característica (P210 ou GND) como a mesma em todos os GA, e de características múltiplas em que, para cada GA, uma mesma característica foi considerada como distinta. O modelo de análise, tanto para P210 quanto para GND, incluiu os efeitos fixos de grupos de contemporâneos e idade da vaca em classes, e os efeitos aleatórios de valores genéticos diretos, genéticos maternos, de ambiente permanente materno e dos efeitos residuais.

$\mathrm{Na}$ forma matricial, o modelo geral pode ser descrito como $y=X \beta+Z_{a} a+Z_{m} m+Z_{p} p+e$, em que: y é o vetor dos caracteres observados; $\beta$, o vetor dos efeitos fixos; a, o vetor dos efeitos genéticos aditivos diretos; $\mathrm{m}$, o vetor dos efeitos genéticos aditivos maternos; $\mathrm{p}$, o vetor do efeito de ambiente permanente materno;

Tabela 1. Número de observações $(\mathrm{N})$, médias $(\bar{x})$ e desvios-padrão (DP) para os grupos ambientais, quanto às características peso à desmama (P210) e ganho de peso do nascimento à desmama (GND).

\begin{tabular}{|c|c|c|c|c|c|c|c|c|c|c|}
\hline \multirow[t]{2}{*}{ Estatística } & \multirow[t]{2}{*}{ P210(kg) } & \multicolumn{4}{|c|}{ Grupos ambientais } & \multirow[t]{2}{*}{ GND (kg) } & \multicolumn{4}{|c|}{ Grupos ambientais } \\
\hline & & P210_MC & P210_MS & P210_FC & P210_FS & & GND_MC & GND_MS & GND_FC & GND_FS \\
\hline$\overline{\mathrm{N}}$ & 18.366 & 4.471 & 4.639 & 4.587 & 4.639 & 17.538 & 4.266 & 4.450 & 4.376 & 4.446 \\
\hline $\bar{x}$ & 221,89 & 225,52 & 231,25 & 213,15 & 217,67 & 181,92 & 184,30 & 190,30 & 174,42 & 178,65 \\
\hline DP & 50,83 & 52,34 & 51,92 & 49,02 & 48,07 & 49,65 & 51,14 & 50,83 & 48,26 & 46,88 \\
\hline
\end{tabular}


$\varepsilon$, o vetor dos efeitos residuais; $X, Z_{a}, Z_{m}$ e $Z_{p}$ são as matrizes de incidência que relacionam $\beta, \mathrm{a}, \mathrm{m}, \mathrm{p}$ à $\mathrm{y}$. Assumiu-se que: $\mathrm{E}[\mathrm{y}]=\mathrm{X} \beta ; \operatorname{Var}(\mathrm{a})=\mathrm{A} \otimes \mathrm{G}_{\mathrm{a}}, \operatorname{Var}(\mathrm{m})=\mathrm{A}$ $\otimes G_{m}, \operatorname{Var}(p)=I_{N m} \otimes G_{p}$ e $\operatorname{Var}(e)=I_{N} \otimes R$, em que, $G_{a}$ é a matriz de covariância genética aditiva; $\mathrm{G}_{\mathrm{m}}$, a matriz de covariância genética aditiva materna; $G_{p}$, a matriz de covariância genética de ambiente permanente materno; $\mathrm{R}$ é a matriz de covariância residual; $\mathrm{A}$, a matriz de parentesco; I, a matriz identidade; $\mathrm{Nm}$, número de mães de animais com registros; N, o número de animais com registros; $\otimes$ é o produto direto entre as matrizes; e que os vetores a, $\mathrm{m}, \mathrm{p}, \mathrm{e} \varepsilon$ são independentes.

Os vetores $\beta$ e a são parâmetros de locação de uma distribuição condicional y| $\beta$, a, m, p. Assumiu-se como priori para $\beta$ uma distribuição uniforme, que reflete um conhecimento prévio vago sobre este vetor. Para o vetor a, assumiu-se como priori uma distribuição normal multivariada, com média 0 e variância $\sigma^{2}{ }_{\mathrm{a}} \mathrm{A}$ e $\mathrm{G}_{\mathrm{a}} \otimes \mathrm{A}$, para as análises uni e multicaracterística, respectivamente. Para $\mathrm{m}$ e $\mathrm{p}$, também se assumiu uma distribuição normal multivariada, com média $0 \mathrm{e}$ variância $\sigma_{m}^{2} A$ e $G_{m} \otimes$ A e variância $\sigma_{p}^{2} I_{e}$ e $G_{p} \otimes I$, para análises uni e multicaracterística, respectivamente.

Para os demais componentes, assumiu-se como priori a distribuição Wishart e qui-quadrado invertida, para análises multi e unicaracterística, respectivamente (Blasco, 2001).

Para as análises uni e multicaracterística, foram geradas cadeias de Gibbs com um milhão de amostras, tendo sido descartadas as primeiras $200 \mathrm{mil}$ e, após estas, uma de cada 20 amostras foi armazenada para inferências sobre a distribuição posterior. A análise da cadeia foi realizada com uso do pacote BOA (Smith, 2007) do programa $R$, para verificar a convergência e dependência entre as amostras, por meio do teste de Heidelberger \& Welch (1983). A importância da interação genótipo $\mathrm{x}$ ambiente foi verificada por meio da correlação genética $\left(r_{g}\right)$. As interações foram consideradas importantes quando os valores de $r_{g}$ entre os GA ficaram abaixo de 0,80 , conforme sugerido por Robertson (1959).

\section{Resultados e Discussão}

As estimativas dos componentes de covariância e variância (Tabela 2) pelos modelos uni e multicaracterística mostraram médias diferentes, tanto do peso à desmama (P210) quanto do ganho de peso do nascimento à desmama (GND). Apesar disto, existe uma área grande de sobreposição das distribuições a posteriori, como observado pelos valores dos intervalos de alta densidade (IAD), o que nos permite dizer que

Tabela 2. Médias e intervalo de alta densidade (IAD) das distribuições posteriores das estimativas de covariância e variância do peso à desmama (P210) e do ganho de peso do nascimento à desmama (GND), nas análises multicaracterística e unicaracterística.

\begin{tabular}{|c|c|c|c|c|c|c|c|c|c|c|c|c|}
\hline \multirow[t]{3}{*}{ Estimativa $^{(1)}$} & \multicolumn{8}{|c|}{ Grupos ambientais da análise multicaracterística } & \multicolumn{4}{|c|}{ Análise unicaracterística } \\
\hline & \multicolumn{2}{|c|}{ P210 estação chuvosa } & \multicolumn{2}{|c|}{ GND estação chuvosa } & \multicolumn{2}{|c|}{ P210 estação seca } & \multicolumn{2}{|c|}{ GND estação seca } & \multicolumn{2}{|r|}{ P210 } & \multicolumn{2}{|c|}{ GND } \\
\hline & Média & IAD $95 \%$ & Média & IAD 95\% & Média & IAD 95\% & Média & IAD 95\% & Média & IAD 95\% & Média & IAD 95\% \\
\hline & \multicolumn{8}{|c|}{ Machos } & \multicolumn{4}{|c|}{ Grupos analisados conjuntamente } \\
\hline$\sigma_{\mathrm{a}}^{2}$ & 293,5 & $171,8-424,2$ & 254,2 & $143,4-370,6$ & 226,4 & $119,8-336,2$ & 170,9 & $82,06-271,5$ & 195,33 & $141,2-251,9$ & 165,33 & $116,6-216,0$ \\
\hline$\sigma_{m}^{2}$ & 134,8 & $66,0-203,4$ & 115,2 & $57,0-173,4$ & 135,4 & $64,83-209,6$ & 137,1 & $75,34-208,2$ & 52,26 & $21,72-84,99$ & 46,35 & $19,70-76,10$ \\
\hline$\sigma_{\mathrm{p}}^{2}$ & 101,2 & $24,3-170,7$ & 94,30 & $45,4-146,6$ & 159,2 & $71,70-242,7$ & 145,1 & $65,67-225,4$ & 92,26 & $55,05-129,2$ & 76,57 & $43,1-110,4$ \\
\hline$\sigma_{\varepsilon}^{2}$ & 880,0 & $763,6-990,8$ & 876,5 & $772,9-986,7$ & 839,9 & $731,9-947,3$ & 816,3 & $711,4-921,8$ & 878,31 & $832,8-922,8$ & 856,37 & $813,3-899,4$ \\
\hline $\mathrm{h}_{\mathrm{a}}^{2}$ & 0,20 & $0,12-0,30$ & 0,19 & $0,10-0,27$ & 0,16 & $0,09-0,24$ & 0,13 & $0,06-0,21$ & 0,16 & $0,11-0,20$ & 0,14 & $0,10-0,18$ \\
\hline $\mathrm{h}_{\mathrm{m}}^{2}$ & 0,09 & $0,046-0,141$ & 0,08 & $0,042-0,127$ & 0,09 & $0,047-0,151$ & 0,10 & $0,058-0,160$ & 0,04 & $0,018-0,069$ & 0,04 & $0,018-0,066$ \\
\hline $\mathrm{p}^{2}$ & 0,07 & $0,018-0,121$ & 0,07 & $0,032-0,107$ & 0,11 & $0,052-0,177$ & 0,11 & $0,049-0,174$ & 0,07 & $0,044-0,104$ & 0,06 & $0,037-0,095$ \\
\hline \multicolumn{13}{|c|}{ Fêmeas } \\
\hline$\sigma_{\mathrm{a}}^{2}$ & 285,9 & $174,9-396,4$ & 240,2 & $139,4-338,4$ & 199,5 & $105,4-298,2$ & 205,3 & $107,8-303,4$ & - & - & - & - \\
\hline$\sigma_{\mathrm{m}}^{2}$ & 122,4 & $57,6-184,1$ & 109,8 & $49,7-163,3$ & 129,7 & $72,27-192,7$ & 114,6 & $59,12-169,0$ & - & - & - & - \\
\hline$\sigma_{\mathrm{p}}^{2}$ & 74,17 & $20,2-146,6$ & 77,81 & $25,5-130,7$ & 119,4 & $62,58-185,6$ & 95,55 & $33,79-165,0$ & - & - & - & - \\
\hline$\sigma_{\varepsilon}^{2}$ & 714,5 & $617,6-815,3$ & 702,4 & $614,4-790,7$ & 620,9 & $532,1-705,4$ & 584,2 & $498,0-668,4$ & - & - & - & - \\
\hline $\mathrm{h}_{\mathrm{a}}^{2}$ & 0,23 & $0,15-0,32$ & 0,21 & $0,12-0,29$ & 0,18 & $0,09-0,27$ & 0,20 & $0,11-0,30$ & - & - & - & - \\
\hline $\mathrm{h}_{\mathrm{m}}^{2}$ & 0,10 & $0,049-0,152$ & 0,09 & $0,046-0,144$ & 0,12 & $0,067-0,176$ & 0,11 & $0,065-0,172$ & - & - & - & - \\
\hline $\mathrm{p}^{2}$ & 0,06 & $0,015-0,120$ & 0,06 & $0,020-0,113$ & 0,11 & $0,057-0,172$ & 0,09 & $0,020-0,113$ & - & - & - & - \\
\hline
\end{tabular}


é pequena a probabilidade de essas estimativas serem diferentes.

A pequena diferença observada entre as estimativas pontuais das variâncias de origem materna, em ambos os sexos nas duas estações, pode ser atribuída à maior disponibilidade de pasto de boa qualidade nas chuvas, bem como às variações na temperatura e umidade e na incidência de endo e ectoparasitas. Assim, ambientes favoráveis tendem a encobrir a diferença genética entre os animais, o que faz com que haja pouca variabilidade nas estimativas. Possivelmente, os bezerros nascidos na época das chuvas tendem a se saciar mais rapidamente pela alta disponibilidade de leite que suas mães oferecem, o que não acontece na época da seca. Portanto, ainda que a estação das chuvas favoreça as diferenças em produção de leite das mães, esta diferença não seria observada nas crias. A real habilidade materna da fêmea pode também ser mascarada pela maior disponibilidade de pastagem para os bezerros, o que diminui a importância e, consequentemente, a variância dos efeitos maternos. Entretanto, na distribuição a posteriori das estimativas de herdabilidade materna (Figura 1), a principal diferença observada ocorreu entre a análise unicaracterística e a análise multicaracterística.

Em ambas as características avaliadas, a maior parte das distribuições a posteriori de herdabilidade direta se sobrepõem. Entretanto, nota-se que a distribuição das estimativas da análise unicaracterística e dos machos na estação seca se afasta das demais. O mesmo comportamento não é observado nas distribuições de herdabilidade materna, pois a única distribuição que não se sobrepõe ao conjunto é a da análise unicaracterística. Segundo Pégolo (2010), o conjunto de genes de uma característica que se expressa em determinado ambiente não é o mesmo que age em outro ambiente. Isto fica mais evidente quando os ambientes possuem diferenças extremas. Assim, quando tais conjuntos de genes são avaliados conjuntamente (análise unicaracterística), o valor da estimativa tende a cair.

Esses resultados mostram a ausência da heterogeneidade de variâncias (Figuras 1 e 2). Com exceção da variância residual em machos e fêmeas na estação das chuvas, houve heterogeneidade de variância, que é uma forma de interação genótipo $\mathrm{x}$ ambiente. Entretanto, deve-se ressaltar que a interação GxA não é necessariamente aliada à presença da heterogeneidade de variâncias, e não é esta a forma de interação que tem maior importância do ponto de vista prático da seleção. A ausência de heterogeneidade, observada no presente trabalho, pode ser decorrente da fase da vida estudada, uma vez que do nascimento até a desmama os animais são menos expostos às variações ambientais, em razão dos cuidados maternos, principalmente da amamentação. Segundo Näsholm (2004), a diferença entre machos e fêmeas aumenta com o avanço da idade, principalmente após a fase de desmama.

Os resultados encontrados no presente estudo corroboram os de Van Vleck \& Cundiff (1998), que concluíram não haver diferenças significativas nas estimativas dos parâmetros genéticos de machos e fêmeas em diversas raças taurinas. Entretanto, Lee \& Pollak (1997) e Stalhammar \& Philipsson (1997) encontraram heterogeneidade de variância entre os sexos, em bovinos Simental, discordante das estimativas encontradas no presente trabalho. Rodriguez-Almeida et al. (1995) e Pégolo (2010) também observaram heterogeneidade de variâncias entre os sexos. Contudo, os autores basearam as suas conclusões em estimativas pontuais, obtidas com o método da máxima verossimilhança restrita.

Apesar da ausência de heterogeneidade de variâncias, a interação GxA foi evidenciada entre todos os GA, nas duas características, quando se avaliaram as correlações genéticas (Figura 2). As médias das distribuições posteriores das correlações genéticas variaram de 0,54 a 0,78 , para P210, e de 0,55 a 0,75 para GND. Da mesma forma que no presente estudo, evidências de interação GxA para características pré-desmama, em diversas raças bovinas, foram relatadas em outros trabalhos (Fridrich et al., 2005; Mascioli et al., 2006; Lopes et al., 2008; Zapata et al., 2010; Espasandin et al., 2011).

A interação GxA no presente estudo indica que grupos de genes, diferentes tanto no P210 quanto no GND, se expressam distintamente entre as estações, mesmo que contribuam de forma semelhante para a variância da característica. Assim, a resposta à seleção para uma característica selecionada em uma estação, nos diferentes sexos, não será semelhante à resposta em outra estação.

Nota-se, pelas distribuições a posteriori (Figura 2) das estimativas das correlações genéticas, que as fêmeas foram as mais afetadas pela mudança das estações. Assim, há um padrão de interpretação diferente quanto aos machos e às fêmeas. 
O menor valor de correlação genética, tanto no P210 quanto no GND (0,54 e 0,55 respectivamente), se dá nas fêmeas, entre estações, o que indica que as fêmeas foram mais sensíveis à mudança da estação das chuvas para a estação seca. Assim, ao realizar a seleção com base nos valores genéticos obtidos por meio das fêmeas, haverá diferenças consideráveis nos animais selecionados para as diferentes estações de nascimento.
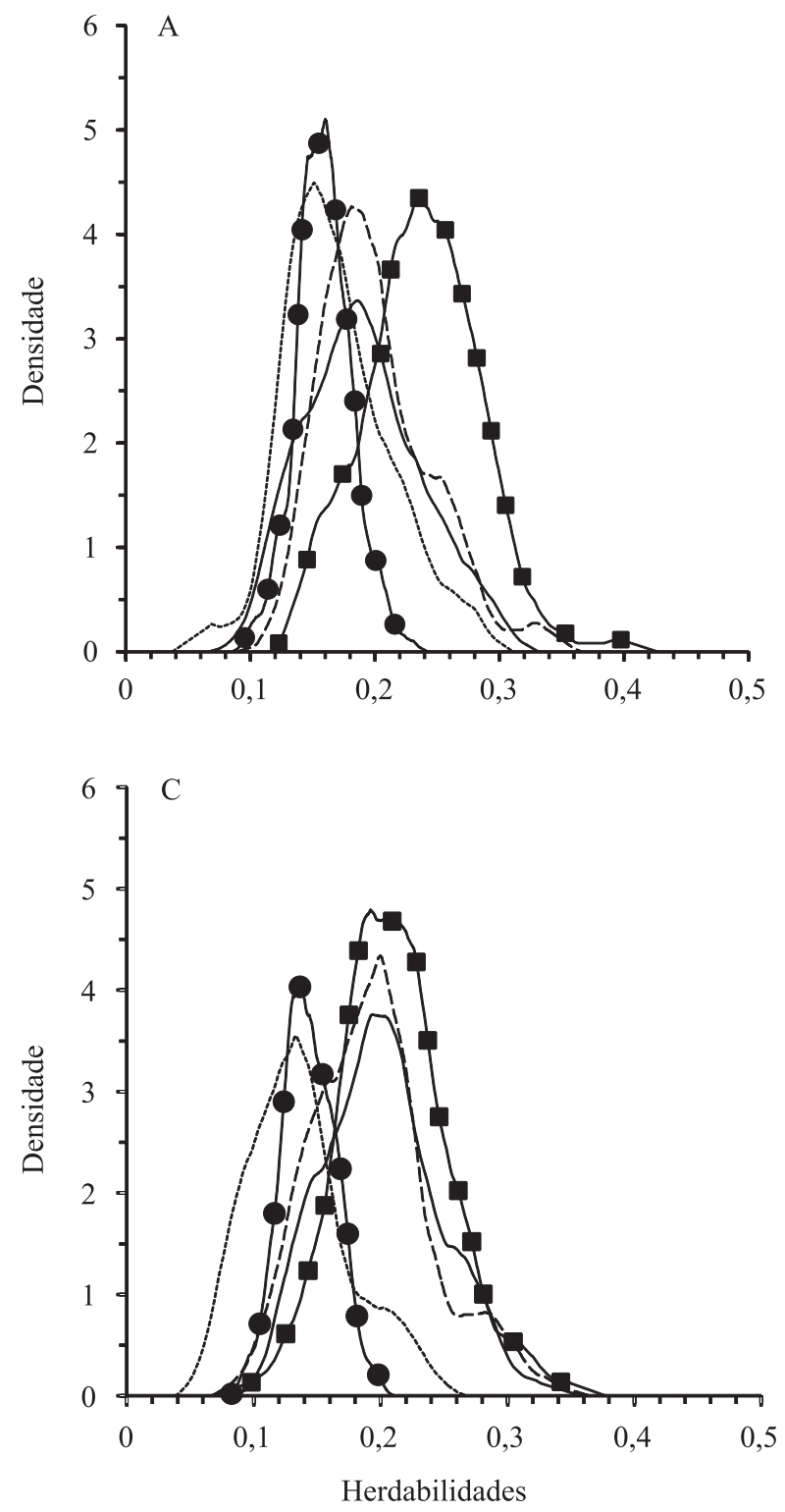

Os maiores valores de correlação genética encontrados no presente trabalho para os machos, entre as duas estações de nascimento, foram de $0,74 \mathrm{e}$ 0,75, para P210 e GND, respectivamente, o que mostra que, para os machos, a mudança dos valores genéticos avaliados nas duas estações seria menor.

Tais resultados podem ser decorrentes de tratamentos preferenciais fornecidos aos machos na estação da seca (suplementação alimentar diferenciada), o que lhes
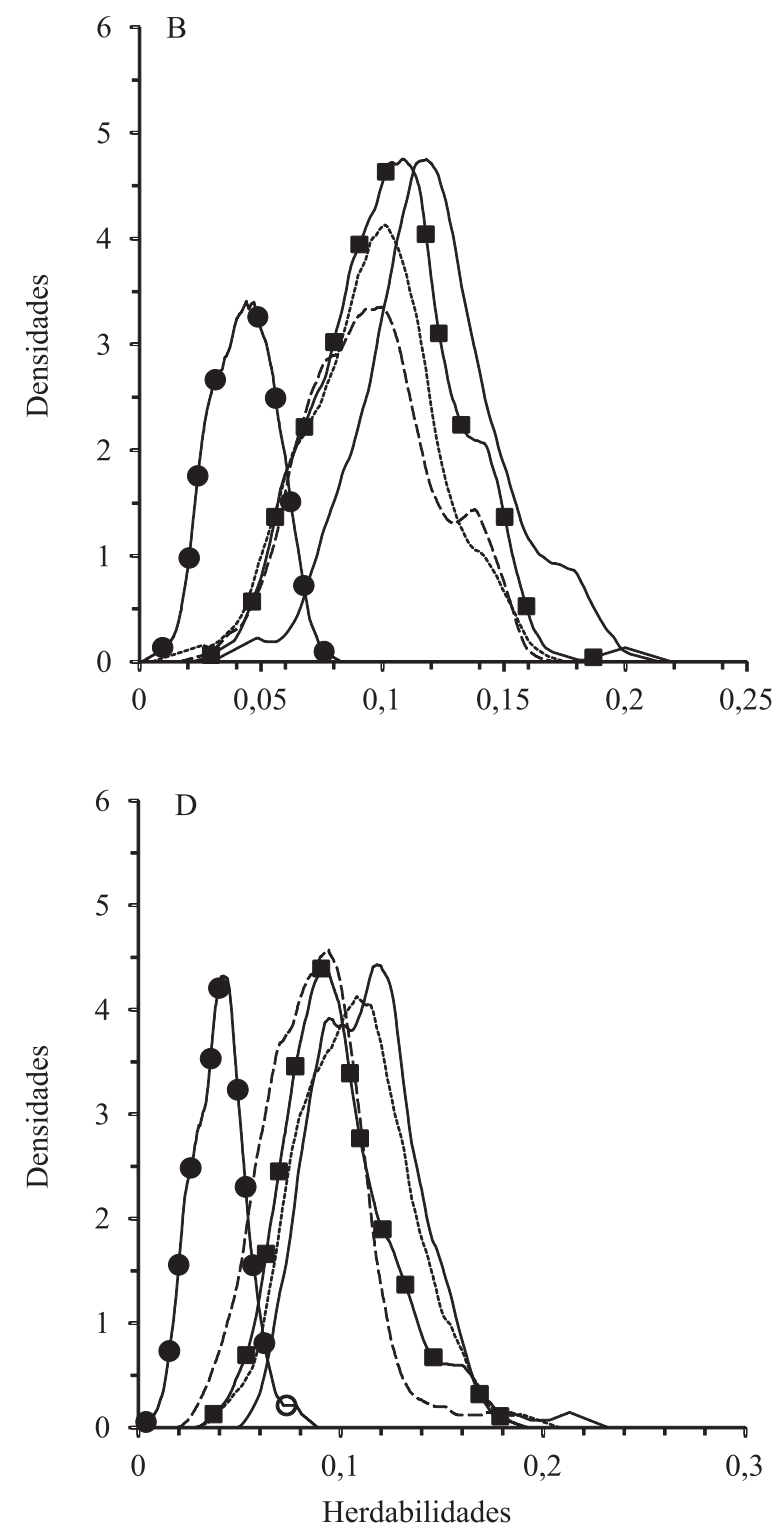

$$
----\cdot \mathrm{M} \_\mathrm{C} \quad \cdots-\cdots \cdot \mathrm{M} S \mathrm{~S} \longrightarrow \mathrm{F}_{-} \mathrm{C} \longrightarrow \mathrm{F}
$$

Figura 1. Densidades a posteriori dos parâmetros genéticos estimados para machos $(\mathrm{M})$ e fêmeas $(\mathrm{F})$, nas estações chuvosa (C) e seca (S), nas análises multicaracterística (M_C) e unicaracterística (UNI): A, herdabilidade direta P210; B, herdabilidade materna P210; C, herdabilidade direta GND; D, herdabilidade materna GND. 
permite manifestar o seu potencial genético de forma mais próxima ao de seu desempenho na estação da chuva. As diferenças na expressão dos genótipos, entre as fêmeas e os machos, parecem terem se acentuado sempre que a estação das secas era incluída nas análises, conforme observa-se pelas seguintes estimativas: fêmeas na estação chuvosa e machos na estação seca (0,64 e 0,63, para P210 e GND, respectivamente), fêmeas na estação seca e machos na estação chuvosa $(0,64$ e 0,68 , para P210 e GND, respectivamente), e fêmeas na estação seca e machos na estação seca $(0,57$ e 0,58, para P210 e GND, respectivamente).
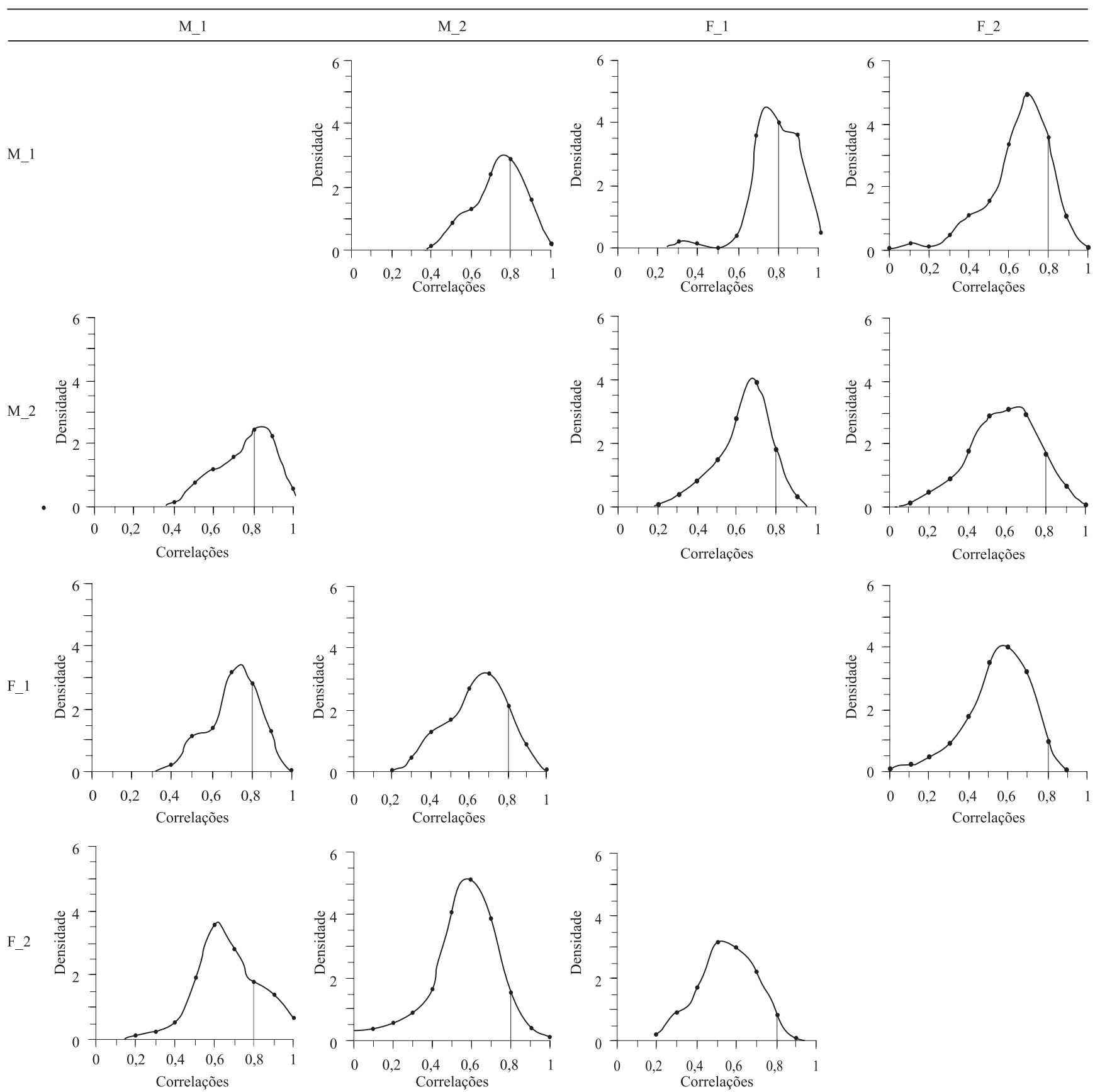

Figura 2. Densidades a posteriori das estimativas das correlações genéticas entre os pesos (acima da diagonal) e ganhos de pesos (abaixo da diagonal), em que a linha demarca o limite quanto à importância da interação genótipo $\mathrm{x}$ ambiente $\left(R_{\mathrm{G}}=0,80\right)$, nas estações chuvosa (C) e seca (S), em machos (M) e fêmeas (F) da raça Simental. 
Quando a correlação genética não incluiu a estação seca, o valor genético das fêmeas se assemelhou, em parte, ao dos machos (correlação genética de 0,78 e 0,70, para P210 e GND, respectivamente), uma vez que a disponibilidade de alimentos de boa qualidade aumenta, o que permite melhor desempenho de ambos os sexos. Tais resultados estão de acordo com o trabalho de Barwick et al. (2009), que estimaram correlações genéticas de diferentes características, em machos e fêmeas nas estações secas e chuvosas, e também verificaram que as correlações entre os sexos eram maiores na estação chuvosa.

A redução percentual nas médias dos valores genéticos dos animais (Figura 3), quando a seleção é baseada em uma análise unicaracterística, comprova as consequências de se ignorar o efeito da interação GxA. A redução foi maior nas fêmeas, tanto para P210 quanto para GND. A perda por ignorar os efeitos da interação
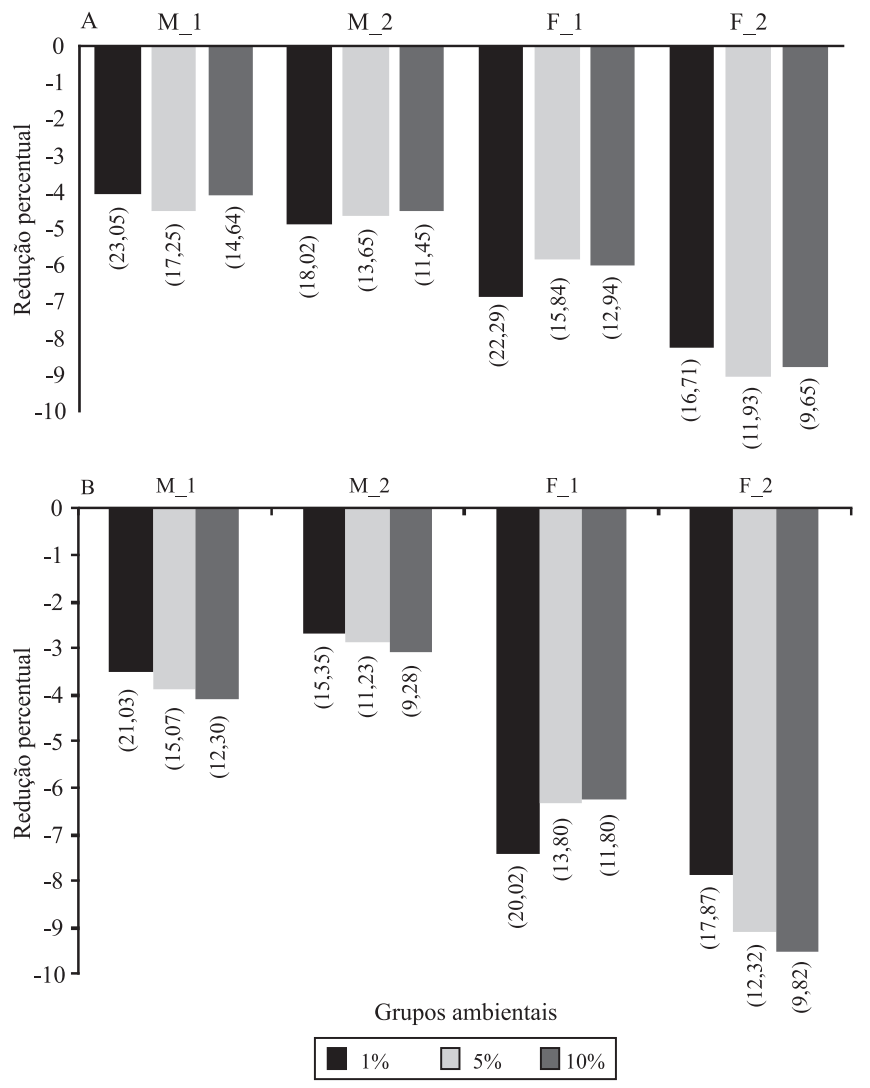

Figura 3. Média dos valores genéticos em análise multicaracterística (entre parênteses), quando selecionados 1,5 e $10 \%$ dos melhores animais, e redução percentual (barras) quando a seleção foi feita com base na análise unicaracterística: A, P210; B, GND. pode chegar a mais de $10 \%$ do valor genético médio dos animais selecionados. As fêmeas apresentaram as maiores reduções na média dos valores genéticos, nas duas estações de nascimento, tendo variado de acordo com a intensidade de seleção. Quanto aos machos, a perda parece ser menos importante. Isto é esperado em razão de as correlações genéticas entre os machos, nos dois ambientes, serem maiores.

As fêmeas foram as mais influenciadas pela presença da interação GxA nas características P210 e GND, pois os valores genéticos obtidos, com e sem a análise com a interação GxA, apresentaram grandes diferenças, o que poderá promover menores taxas de ganhos pela seleção. Entretanto, como nas fêmeas a intensidade de seleção possível é muito pequena, a interferência efetiva da interação GxA no ganho genético pode ser desprezada. Assim, não é necessário que essas diferenças sejam consideradas na avaliação genética dos animais.

\section{Conclusão}

Há efeito das estações de nascimento nas características avaliadas, em todos os grupos ambientais, e a interação GxA é mais evidente em fêmeas do que em machos.

\section{Agradecimentos}

À Fundação de Amparo à Pesquisa do Estado de São Paulo, pelo apoio financeiro.

\section{Referências}

BARWICK, S.A.; JOHNSTON, D.J.; BURROW, H.M.; HOLROYD, R.G.; FORDYCE, G.; WOLCOTT, M.L.; SIM, W.D.; SULLIVAN, M.T. Genetics of heifer performance in "wet" and "dry" seasons and their relationships with steer performance in two tropical beef genotypes. Animal Production Science, v.49, p.367-382, 2009

BLASCO, A. The Bayesian controversy in animal breeding. Journal of Animal Science, v.79, p.2023-2046, 2001.

ESPASANDIN, A.C.; URIOSTE, J.I.; CAMPOS, L.T.; ALENCAR, M.M. de. Genotype $\times$ country interaction for weaning weight in the Angus populations of Brazil and Uruguay. Revista Brasileira de Zootecnia, v.40, p.568-574, 2011

FRIDRICH, A.B.; SILVA, M.A.; FRIDRICH, D.; CORRÊA, G.S.S.; SILVA, L.O.C.; SAKAGUTI, E.S.; FERREIRA, I.C.; VALENTE, B.D. Interação genótipo $\times$ ambiente e estimativas de parâmetros genéticos de características ponderais de bovinos Tabapuã. Arquivo Brasileiro de Medicina Veterinária e Zootecnia, v.57, p.663-672, 2005. 
HEIDELBERGER, P.; WELCH, P.D. Simulation run length control in the presence of an initial transient. Operations Research, v.31, p.1109-1144, 1983

JONG, G. de; BIJMA, P. Selection and phenotypic plasticity in evolutionary biology and animal breeding. Livestock Production Science, v.78, p.195-214, 2002.

KOLMODIN, R.; STRANDBERG, E.; MADSEN, P.; JENSEN, J.; JORJANI, H. Genotype by environment interaction in nordic dairy cattle studied using reaction norms. Acta Agriculturae Scandinavica Section A - Animal Science, v.52, p.11-24, 2002.

LEE, C.; POLLAK, E.J. Influence of partitioning data by sex on genetic variance and covariance components for weaning weight in beef cattle. Journal of Animal Science, v.75, p.61-67, 1997.

LOPES, J.S.; RORATO, P.R.N.; WEBER, T.; BOLIGON, A.A.; COMIN, J.G.; DORNELLES, M. de A. Efeito da interação genótipo $\mathrm{x}$ ambiente sobre o peso ao nascimento, aos 205 e aos 550 dias de idade de bovinos da raça Nelore na Região Sul do Brasil. Revista Brasileira de Zootecnia, v.37, p.54-60, 2008.

MASCIOLI, A. dos S.; ALENCAR, M.M. de; FREITAS, A.R. de; MARTINS, E.N. Estudo da interação genótipo $\times$ ambiente sobre características de crescimento de bovinos de corte utilizandose inferência bayesiana. Revista Brasileira de Zootecnia, v.35, p.2275-2284, 2006.

MISZTAL, I. BLUPF90 family of programs. 2009. Available at: $<$ http://nce.ads.uga.edu/ ignacy/newprograms.html $>$. Accessed on: 10 Jan. 2010.

NÄSHOLM, A. Influence of sex on genetic expressions and variance of 4-month weight of Swedish lambs. Livestock Production Science, v.86, p.137-142, 2004.
PÉGOLO, N.T. Interação genótipo-ambiente e sensibilidade ambiental em bovinos de corte. 2010. 116p. Tese (Doutorado) Universidade de São Paulo, Ribeirão Preto.

ROBERTSON, A. The sampling variance of the genetic correlation coefficient. Biometrics, v.15, p.469-485, 1959.

RODRIGUEZ-ALMEIDA, F.A.; VAN VLECK, L.D.; CUNDIFF, L.V.; KACHMAN, S.D. Heterogeneity of variance by sire breed, sex, and dam breed in 200- and 365-day weights of beef cattle from a top cross experiment. Journal of Animal Science, v.73, p.2579-2588, 1995.

SAS INSTITUTE. SAS/STAT: user's guide: version 9.1.3. Cary: SAS Institute, 2008.

SMITH, B.J. boa: An R package for MCMC output convergence assessment and posterior inference. Journal of Statistical Software, v.21, 2007. Available at: <http://www.jstatsoft.org/v21/ i11/paper>. Accessed on: 10 Jan. 2010.

STALHAMMAR, H.; PHILIPSSON, J. Sex-specific genetic parameters for weaning and post-weaning gain in Swedish beef cattle under field conditions. Acta Agriculturae Scandinavica Section A - Animal Science, v.47, p.138-147, 1997.

VAN VLECK, L.D.; CUNDIFF, L.V. Sex effects on breed of sire differences for birth, weaning, and yearling weights. Journal of Animal Science, v.76, p.1528-1534, 1998.

WEST-EBERHARD, M.J. Developmental plasticity and evolution. New York: Oxford University, 2003. 794p.

ZAPATA, E.M.A.; MUÑOZ, M.F.C.; TORRES, J.M.C.; GARAY, O.D.V. Genotype-environment interaction in multibreed bovine populations in the Colombian low tropic. Revista Colombiana de Ciencias Pecuarias, v.23, p.145-157, 2010.

Recebido em 27 de setembro de 2010 e aprovado em 18 de fevereiro de 2011 